\title{
Performance Analysis of a COFDM/FM In-band Digital Audio Broadcasting System
}

\author{
Pascal Scalart, Michel Leclerc*, Paul Fortier, and Huu Tuê Huynh \\ Department of Electrical and Computer Engineering, Laval University, Québec, Canada G1K 7P4 \\ *Cybectec, 8165 rue du Mistral, suite 100, Charny, Québec, Canada G6X 3R8
}

\begin{abstract}
In this paper, we present a performance analysis of a Coded Orthogonal Frequency Division Multiplexing DAB systems that is power multiplexed over a commercial FM station. We model the mobile channel with a time-frequency scattering function. Results show that the performance of the FM demodulator is crucial, since the level of the residual FM signal has a strong influence on the BER performance of the COFDM system. As with other mobile communication systems, the use of interleaving greatly enhances the performances.
\end{abstract}

\section{INTRODUCTION}

Traditionally, three types of multiplexing methods have been used in communication systems, namely in time (TDMA), in frequency (FDMA) and in code (CDMA). These three methods have been extensively studied and are implemented or planned to be implemented in several communications systems. However, there is a fourth method, proposed several years ago, that has received little attention and has not been implemented yet, mostly due to practical reasons: power multiplexing. In power multiplexing, the capture effect of FM demodulation is used to separate signals that are at different power levels. It is well known that the capture effect in FM transmission allows the demodulation of the stronger signal without noticeable interferences from the weaker one [1], [2]. Using this principle, it is possible to realize a power multiplexing system that first demodulates the stronger FM signal, remodulates it and subtracts it from the incoming signal. The resulting signal, containing only the weaker FM signal, is then demodulated. This method has been proposed for the multiplexing of Digital Audio Broadcasting (DAB) and FM signals [3].

With the advent of VLSI circuits and advanced digital communications techniques, it is now possible to broadcast compact disk (CD) quality programs. One of the systems proposed for DAB uses a modulation technique called Coded Orthogonal Frequency Division Multiplexing (COFDM). At the WARC 92 conference, it was decided to allocate a $40 \mathrm{MHz}$ frequency band at $1.5 \mathrm{GHz}$ for DAB services. However, some groups in the United States are proposing to transmit the DAB signal in the FM broadcasting band. Moreover, the DAB signal would be transmitted through power multiplexing over an existing FM station, thus saving money to the broadcasters.

In this paper, we present a performance analysis of a Coded Orthogonal Frequency Division Multiplexing DAB system that is power multiplexed over a commercial broadcasting FM station. We are interested in the Bit Error Rate (BER) performance of the COFDM system in the presence of Additive White Gaussian Noise (AWGN), residual FM signal (due to imperfections in the power multiplexing demodulation scheme), and multipath fading (due to the mobility of the receiver). The mobile radio channel was modeled by a time-frequency scattering function. We have found that the performance of the FM demodulator is crucial, since the level of the residual FM signal has a strong influence on the BER performance of the COFDM system. Moreover, the use of interleaving greatly enhances the performance, as it is the case with other mobile communication systems.

The structure of the paper is as follows. In Section II, the transmission system is described. The in-band FM power multiplexing system is presented, followed by a more detailed description of the COFDM technique and the way it was simulated. Section III deals with the simulation of the mobile radio channel. In Section IV, the results of our simulations are presented and discussed, first for the AWGN channel, and then for the mobile radio channel. The paper concludes with Section V.

\section{TRANSMISSION SYSTEM}

The transmission system studied in this paper is shown in Fig. 1. The digital COFDM signal, $E_{C O F D M}(t)$, is transmitted on the same analog channel used by the FM broadcast signal, $E_{F M}(t)$. The two signals are power multiplexed, with an FM to COFDM power ratio of $30 \mathrm{~dB}$, typically. This is required for compatibility; the COFDM signal must not interfere with conventional FM broadcasting reception.

At the receiver, the two signal are present. In order to demodulate the COFDM signal, it is first necessary to demodulate the FM signal. Due to the capture effect, the COFDM signal will not be present at the output of the FM demodulator. The demodulated FM signal is then remodulated and subtracted from the incoming signal. We are then left with the COFDM signal, $S_{\text {COFDM }}(t)$, additive noise $n(t)$ with spectral density $N_{0} / 2$ and a residual FM signal, $R_{F M}(t)$, due to imperfection in the power multiplexing scheme. The signal at the input of the COFDM demodulator is thus given by 


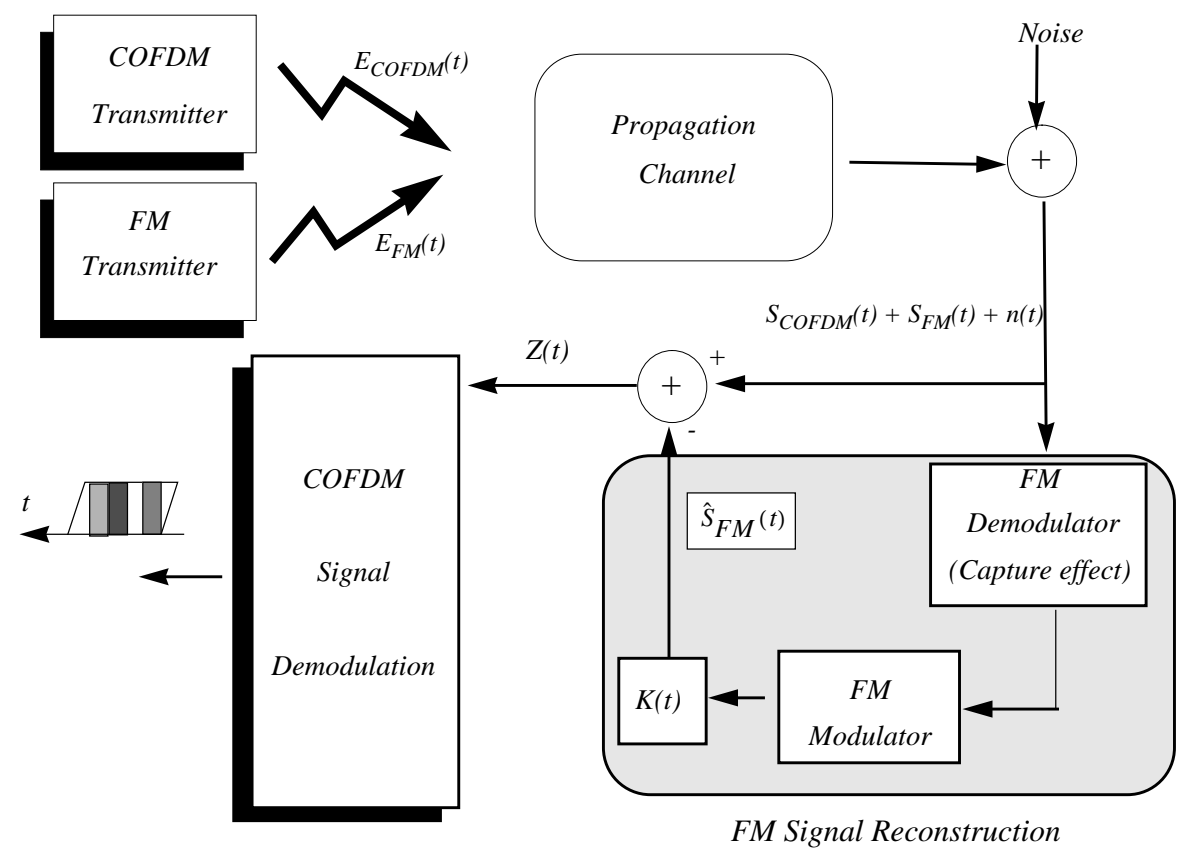

Fig. 1. Transmission system.

$$
Z(t)=S_{C O F D M}(t)+R_{F M}(t)+n(t)
$$

with

$$
R_{F M}(t)=S_{F M}(t)-\hat{S}_{F M}(t)
$$

where $\hat{S}_{F M}(t)$ represents the remodulated FM signal coming from the capture effect receiver.

\section{A. COFDM Transmission Technique}

Initially proposed for the European DAB system [4], COFDM is a parallel transmission scheme. In this type of communication system, the information to be transmitted is separated into $N$ regularly spaced orthogonal sub-carriers, each having the same transmission rate (see Fig. 2). The elementary symbol interval, $T_{U}$, is therefore increased by a factor of $N$, thus reducing the sensitivity of the system to Inter-Symbol Interference (ISI) introduced by the transmission channel.

In order to protect the data against additive noise and deep fades, redundancy is introduced in the form of a convolutional code with rate $R=1 / 2$ and constraint length $L=$ 7 (64 states). The use of such a technique in a multi-carrier system introduces a correlation between transmitted signals on the sub-carriers and allows for the recuperation of the data on a severely distorted sub-carrier from the data on adjacent sub-carriers.

After coding, the data stream is separated into $N$ substreams, each one modulating a particular sub-carrier at frequency $f_{k}=f_{0}+k \Delta F, k=1, \ldots, N$. The choice of the frequency spacing $\Delta F=1 / T_{u}$ guarantees that the sub-carriers are orthogonal over the symbol time $T_{u}$. The modulation and demodulation operations can be performed using FFT techniques, thus reducing the complexity of implementation.

The application that is envisioned for the in-band broadcasting system is the transmission of a CD quality signal. With existing compression techniques, a rate of $256 \mathrm{kbits} / \mathrm{s}$ is needed for stereo sound. Given the spectral efficiency that is required, a DQPSK modulation scheme was used on the sub-carriers.

In order to mitigate the effect of IIS on the system, a guard interval of length $T_{g}$ in placed at the beginning of each symbol. During the guard interval, no information is transmitted. It is necessary to choose the time $T_{g}$ so that it is larger that the length $T_{m}$ of the impulse response of the transmission channel. However, the total length of a symbol, $T_{s}=T_{g}+T_{u}$, must not be too large compared to the time variation of the channel (characterized by the maximum Doppler frequency $v_{m}$ ). The symbol duration must therefore satisfy the following double inequality:

$$
\frac{1}{F_{c}} \ll T_{s} \ll T_{c}
$$

where $T_{c}=1 / v_{m}$ is the coherence time and $F_{c}=1 / T_{m}$ is the coherence frequency band of the channel. For a mobile radio channel, the maximum frequency deviation is given by $v_{m}=f_{0} V / c$, where $c$ is the speed of light, $V$ is the speed of the vehicle (supposed constant) and $f_{0}$ is the carrier frequency.

For the particular application studied in this work (commercial FM band), the maximum frequency deviation for a vehicle moving at a speed of $120 \mathrm{~km} / \mathrm{h}$ is $12 \mathrm{~Hz}$. In order to satisfy the conditions given in (3), we have fixed the guard 


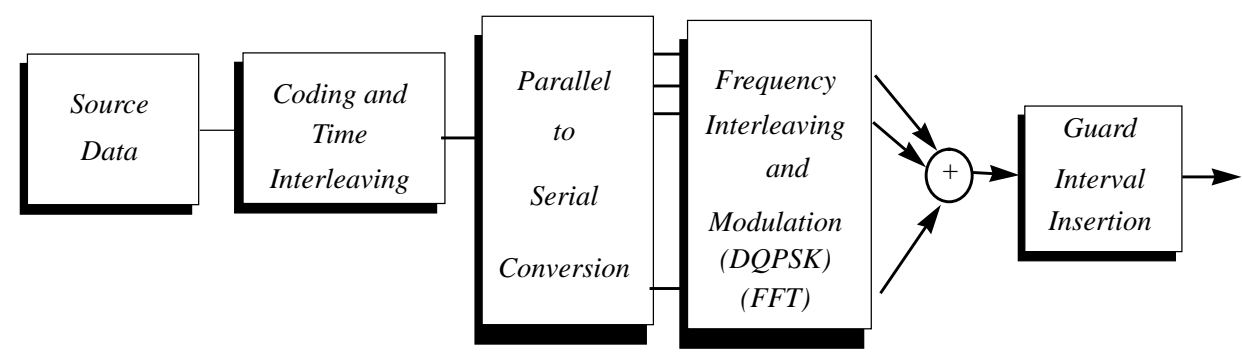

(a) COFDM transmission system

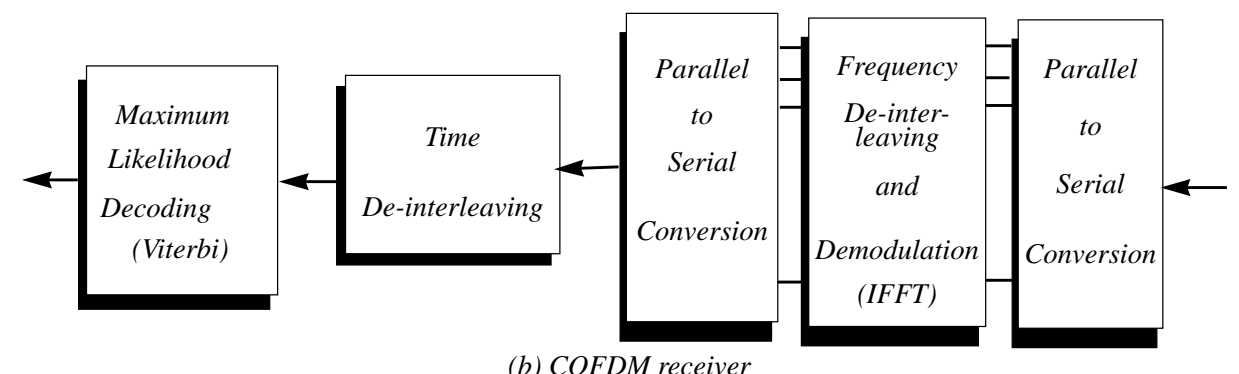

Fig. 2. Transmission and reception principles.

interval, $T_{g}$, at $15.565 \mu$ s (according to the average scattering characteristics of the mobile radio channel) [5], and the total symbol duration, $T_{u}$, at $664.12 \mu \mathrm{s}$. The use of a guard interval introduces a power loss of about $0.1 \mathrm{~dB}$ with respect to a system without one, which can be considered negligeable. To transmit at a data rate of $256 \mathrm{kbits} / \mathrm{s}$, the number of sub-carriers, $N$, must be equal to 174 . The total efficiency of the system is therefore $0.977 \mathrm{bit} / \mathrm{s} / \mathrm{Hz}$ for a total bandwidth of $262 \mathrm{kHz}$. This is a reasonable value giving the frequency spacing between adjacent stations in commercial FM.

This transmission system fully uses interleaving techniques, both in the frequency (pseudo-random interleaver) and in the time (block interleaver) domains. These techniques reduce the correlation between adjacent signal samples at the input of the decision device. As we will see later, interleaving greatly improves the performances of the system, especially at high signal to noise ratios.

The receiver simply realizes the inverse operations of the transmitter. After demodulation (using an FFT) and deinterleaving, the signal is sent to the decoder and the decision device. The maximum likelihood detection is done using the Viterbi algorithm with 3-bit soft decisions and a path memory length that is 5 times the constraint length of the code.

\section{B. Simulation of the COFDM System}

It is very difficult to analyze directly a communication system such as the one described above. Its performances are a function of the quality of the FM receiver, notably the ability of the receiver to produce a residual FM signal, $R_{F M}(t)$, with an average power that is small compared to the power of the COFDM signal. Without precise informa- tions on the performances of the receiver, such as the $R_{F M}(t)$ to $S_{C O F D M}(t)$ power ratio or the spectral characteristics of the residual FM signal, an interesting approach is to evaluate the overall performance of the system using a model for the residual FM signal.

To do this, we have made the supposition that the residual FM signal has spectral characteristics similar to an ordinary FM signal. With this hypothesis, we have found in a prior study [5] that the performance of the system did not depend strongly on the structure of the residual FM signal and that a model based on two modulating sinusoids was sufficient. We will therefore consider in this study that the interfering FM signal corresponds to a signal having two low frequency modulating signals $\left(f_{m 1}=15 \mathrm{kHz}\right.$ and $f_{m 2}=$ $30 \mathrm{KHz}$, with $\beta=5$ ). These values are chosen so that the bandwidth of the FM signal is $200 \mathrm{kHz}$, corresponding to commercial broadcasting FM.

\section{Simulation of the Mobile Radio Channel}

It is better to represent narrow-band real signals using their complex envelopes. Usually, the relation between these two quantity is written as

$$
x(t)=\operatorname{Re}\left[x_{e}(t) e^{j 2 \pi f_{o} t}\right]
$$

where $R e[$.$] is the real part of the quantity, x_{e}(t)$ is the complex envelope of $x(t)$ and $f_{0}$ is the carrier frequency.

Generally, the characterization of the mobile radio propagation channel can be realized using a general description based on time-varying linear systems. The variations of the propagation channel can be described from systems taking into account the actual physical mechanisms of propagation. 
Since the channel is time-varying, its characteristics can be represented as a two dimensional function, $h(t, \tau)$. The complex envelope of the signal is therefore given by a generalized convolution integral

$$
r_{e}(t)=\int x_{e}(t-\tau) h(t, \tau) d \tau
$$

The temporal variations of $h(t, \tau)$ depend on the speed of the vehicle, the wavelength of the transmitted signal and the characteristics of the scattering processes. Thus, the impulse response incorporates all the relations linking the influence of the channel on the transmitted signal with the physical environment specific to the propagation.

\section{A. Model of the Propagation Channel}

In order to obtain a complete statistical description of the channel, we will adopt a partition scheme based on "elementary zones" corresponding to a small geographical area or a short time duration (on the order of a few wavelengths) with respect to the slow variations of the channel. Thus, the average value of the received signal virtually appears to be constant and the channel can be considered to be wide sense stationary (WSS). Furthermore, the signals coming from the different scatterers, having different propagation delays, can be considered to be uncorrelated.Therefore, the two dimensional precess can be approximated by a wide sense stationary uncorrelated scatterers (WSSUS) process [6], [7]. The time-frequency correlation function is thus given by

$$
R(\Delta f, \Delta t)=E\{H(f, t) \times H(f+\Delta f, t+\Delta t)\} .
$$

The propagation channel described in the above manner is constituted of several uncorrelated scatterers in the propagation/Doppler frequency deviation domain. The channel is thus perfectly described using the time-frequency power spectral density

$$
S(\tau, v)=\iint R(\Delta f, \Delta t) e^{j 2 \pi \Delta f \tau} e^{-j 2 \pi \nu \Delta t} d(\Delta f) d(\Delta t) .
$$

This function allows the complete characterization of the propagation conditions caused by a given physical environment. The power spectral density is strictly non-negative definite and it can be decomposed as follows:

$$
P(\tau, v)=V(\tau, v) \times V^{*}(\tau, v) .
$$

A technique that is often employed in spectral analysis is to evaluate the transfer function of the channel from the power spectral density using

$$
H(f, t)=\iint V(\tau, v) e^{-j 2 \pi f \tau} e^{j 2 \pi v t} d \tau d v .
$$

To evaluate the transfer function, it is necessary to have a model for the power spectral density $P(\tau, v)$. Several models have been proposed in the literature. We have used the approach given in [8] where it is shown that $P(\tau, v)$ can be given by the following second order non-negative function, with average $S(\tau, v)$ :

$$
P(\tau, \nu)=|r(\tau, \nu)|^{2} \times S(\tau, \nu)
$$

where $r(\tau, v)$ is a unit variance, zero average, Rayleigh distributed, complex bi-variate process. This process could also include a line-of-sight coherent component.

To reconstitute the transfer function $H(f, t)$ given in (9), we must know the scattering function $S(\tau, v)$ given by (7). Thus, the method to generate the time-varying transfer function takes into account the principal mechanisms of the physical processes using a few statistical functions. In fact, $S(\tau, \nu)$ is perfectly defined from the knowledge of the Doppler and the group delay spread power spectra. We will now describe the models that we have used for these two functions.

\section{B. Models Used}

The time variations of the phase of the received signal coming from a given scatterer $D_{i}$ are caused by the movement of the receiving antenna. If we consider the scatterer to vehicle distance to be large and the speed of the vehicle, $V$, to be constant, the instantaneous phase of the received signal is given by

$$
\varphi^{i}(t)=\varphi_{o}^{i}+\frac{2 \pi}{\lambda} V t \cos \left(\alpha_{i}\right)
$$

where $\phi_{0}^{i}$ is a constant phase at the origin and $\alpha_{i}$ is the relative angle between the incident wave coming from scatterer $D_{i}$ and the direction of the vehicle. Thus, the received signal is affected by a Doppler frequency deviation with a maximal value given by $v_{m}=V / \lambda$.

Considering that the vehicle has an omnidirectional receiving antenna and that the scatterers are regularly spaced with respect to the vehicle (urban area), it is possible to show that the Doppler power spectrum $\gamma(v)$ of the vertically polarized component of the electrical field can be written as [9]

$$
\gamma(v)=\frac{\sigma^{2}}{\pi} \times \frac{1}{\sqrt{v_{m}^{2}-v^{2}}}
$$

which implies the following correlation function:

$$
\psi(t)=\sigma^{2} \times J_{o}\left(2 \pi v_{m} t\right)
$$

where $J_{0}($.$) is the Bessel function of the first kind.$

When there is a direct path between the transmitter and the receiver (sub-urban area), the Doppler power spectrum is modified with the addition of a component whose position in the spectrum is a function of the relative angle between the direct path and the direction of the vehicle.

The group delay spread characteristics for VHF and UHF mobile radio channels have been studied extensively 
[10], [11]. However, different configurations have been observed given the structure of the environment that was studied. In our study, we used two models, corresponding respectively to an urban and a sub-urban environment.

For urban areas where there is no direct path between the transmitter and the receiving antenna, studies on the characterization of the power distribution with respect to group delay spread [12], $P(\tau)=S(\tau, 0)$, have shown that they are well represented by a Maxwell distribution

$$
P(\tau)=\frac{\tau^{2} \sqrt{2}}{\Delta^{3} \sqrt{\pi}} \times \exp \left[-\frac{\tau^{2}}{2 \Delta^{3}}\right] \text { for }(\tau \geq 0)
$$

where $\Delta=\frac{D \sqrt{\pi}}{\sqrt{3 \pi-8}}$, with $D$ being the group delay spread of the impulse response.

For sub-urban areas, where there is a direct path between the transmitter and the receiving antenna, the power distribution as a function of the group delay spread is given by [9], [13]

$$
P(\tau)=\frac{1}{D} \times \exp \left[-\frac{\tau}{D}\right] \text { for }(\tau \geq 0) .
$$

The propagation channel modeled as above satisfies all conditions for wide sense stationarity over a given time interval. The non-stationary behavior is taken into account in two ways. First, by periodically evaluating the power spectral density $P(\tau, v)$ given by (10). Second, by superimposing a long term slowly time-varying signal characterized by a log-normal probability density.

\section{Performance Analysis of the Transmission SYSTEM}

Monte Carlo simulations were used to evaluate the Bit Error Rate (BER) performances of the system. A total of 600000 binary symbols were generated for each simulation runs. This number guaranteed a relative precision of 0.2 for a BER of the order of $10^{-4}$ on an Additive White Gaussian Noise Channel, with a confidence interval of $90 \%$. In every simulation run, we considered that the vehicle was moving at a constant speed of $60 \mathrm{~km} / \mathrm{h}$ and that within the time of the simulation, the vehicle moved by $25 \lambda$, where $\lambda$ is the carrier wavelength. This allowed us to consider several configuration for error bursts at the receiver. Thus the effect of ISI, due to time and frequency variations in the channel, were taken into account.

\section{A. Additive White Gaussian Noise Channel}

We first looked at the performance of the system in an AWGN channel. This channel does not introduce distortions on the transmitted signals. Therefore, at the input of the COFDM demodulator we find the useful signal, the residual FM signal and the additive noise (see Fig. 1). We have evaluated the BER as a function of the signal to noise ratio (i.e. average COFDM signal power over average noise power) for several values of FM to COFDM signal power ratios. These results will serve as references when we want to calculate the degradation due to fading. The results are shown on Fig. 3. The average noise power was computed using a bandwidth equal to the bandwidth of the COFDM signal, i.e. $N / T_{u}$.

We can already observe the irreducible BERs that exist even for large (>15 dB) signal to noise ratios and for FM to COFDM power ratios larger than $-12 \mathrm{~dB}$. This can be explained by the fact that, in the frequency domain, certain components of the residual FM signal interfere with certain sub-channels of the COFDM system, thus increasing the BER on these particular channels.

\section{B. Mobile Radio Channel}

For the mobile radio channel, we will show results for the urban area case. The channel was simulated by extending the model described in Section III to the discrete time case using two dimensional DFTs. The average delay spread, $D$, was fixed at $2.1 \mu \mathrm{s}$, although the maximum value for the spread of the impulse responses could be $10 \mu \mathrm{s}$. The carrier frequency was set at $100 \mathrm{MHz}$. With a vehicle speed of $60 \mathrm{~km} / \mathrm{h}$, the maximum frequency offset is $6 \mathrm{~Hz}$.

Fig. 4 shows the time-frequency variations of the channel, i.e. the modulus of the time-varying transfer function $H(f, t)$, over a period of 0.7 second. As we can see, the subchannels of the COFDM system will be affected differently (frequency selective fading) and this selectivity is timevarying.

A typical temporal variation of the received signal is given in Fig. 5, for a time duration corresponding to a simulation run. As we can see, the envelope of the received signal suffers deep fades, up to 25 to $30 \mathrm{~dB}$, with respect to its average value. The envelope of the signal can be characterized by a Rayleigh probability density.

An example of fading is given in Fig. 6. The transfer function is characterized by a constant attenuation $A$ and by

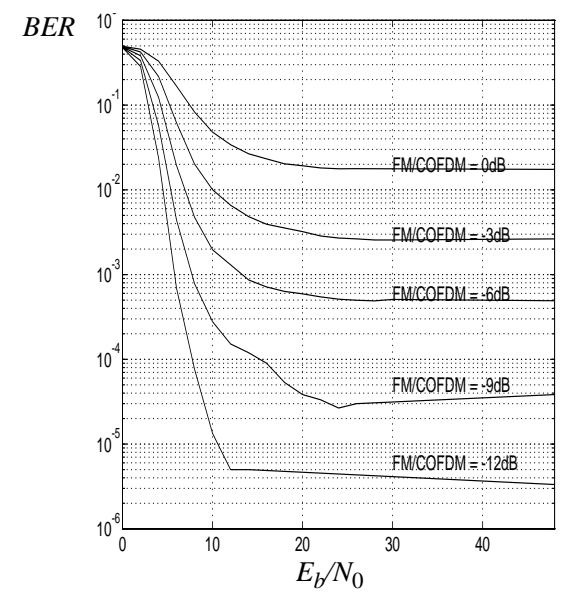

Fig. 3. Performances of the system in an AWGN channel. 
fadings having a maximum relative depth $B$ of about $30 \mathrm{~dB}$ with respect to $A$. Some of the COFDM sub-channels will therefore experience strong attenuations, which will increase the BER on these particular sub-channels.

Fig. 7 shows the time localization of the errors for a value of $E_{b} / N_{0}$ of $18 \mathrm{~dB}$ and for an FM to COFDM power ratio of $-21 \mathrm{~dB}$. Each vertical line corresponds to the number of errors observed at the output of the decoder over a

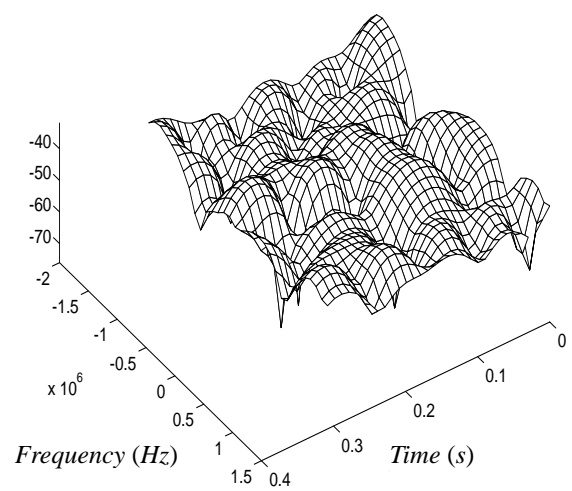

Fig. 4. Time-varying transfer function.

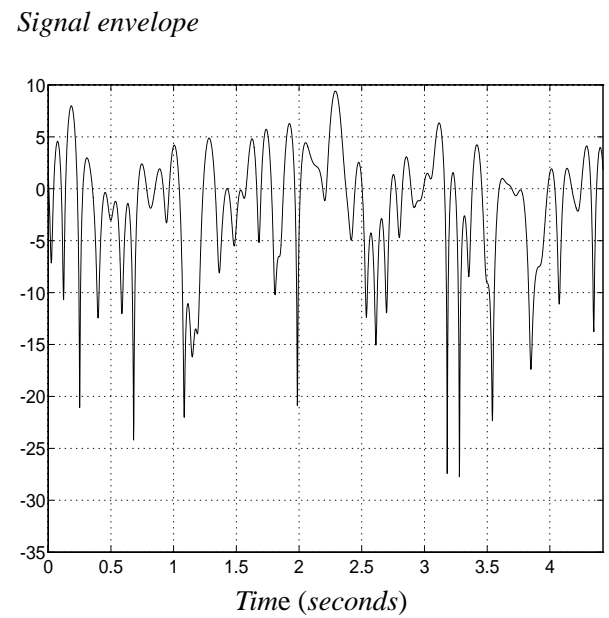

Fig. 5. Time variations of the envelope of the signal.

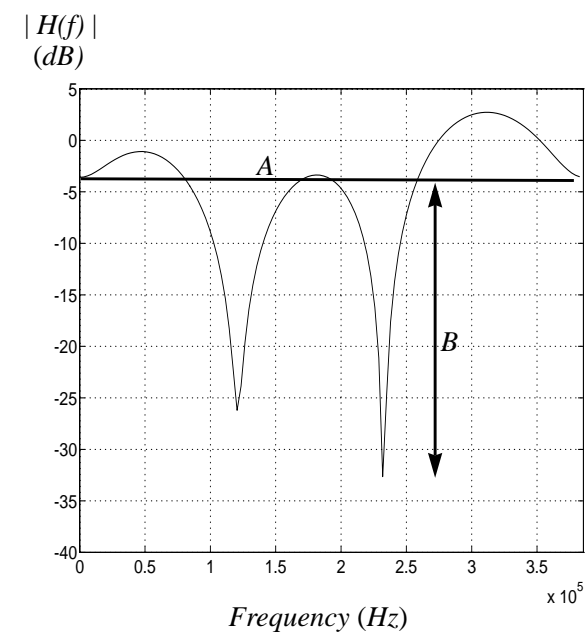

Fig. 6. Time variations of the envelope of the signal.
$0.4 \mathrm{~ms}$ time frame. We can observe that, for such a propagation channel, the signals at the input of the Viterbi decoder are not perfectly uncorrelated and that therefore the errors at the output of the decoder are bursty. If we compare Fig. 7 with Fig. 5, we can see that the errors are mostly produced during deep fade periods.

The average BER curves, as a function of $E_{b} / N_{0}$ and for different values of FM to COFDM power ratios, are shown in Fig. 8. With these curves, we can study the irreducible BER corresponding to large signal to noise ratios (asymptotic performances). As expected, the irreducibles BERs are more important when fading is present, for a given FM to COFDM power ratio. This can be explained by the local BER degradation of the sub-carriers affected by deep fades.

The analysis of these results shows that interleaving is very important in the overall performances of the system. If we study the system without interleaving (Fig. 9), we can clearly see a degradation of the performances. However, interleaving introduces an additional delay in the communication chain. In order to decrease this delay, a partial time interleaving can be done. In this case, the successive symbols at the input of the decoder are not completely indepen-

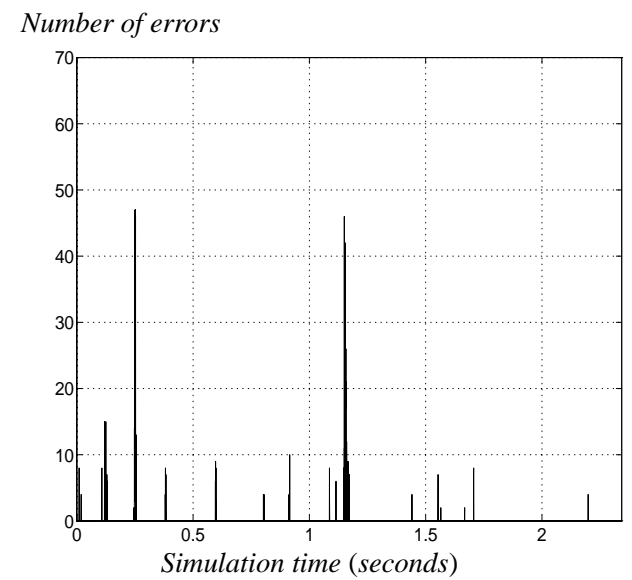

Fig. 7. Error positions in time.

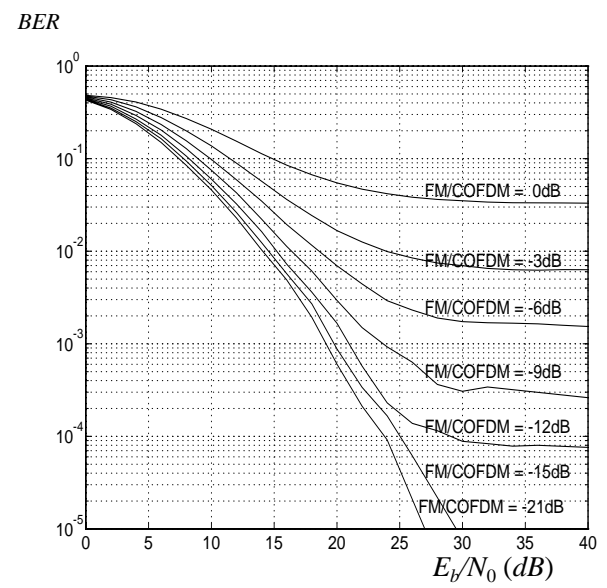

Fig. 8. Performances of the system in a mobile radio channel (with interleaving). 


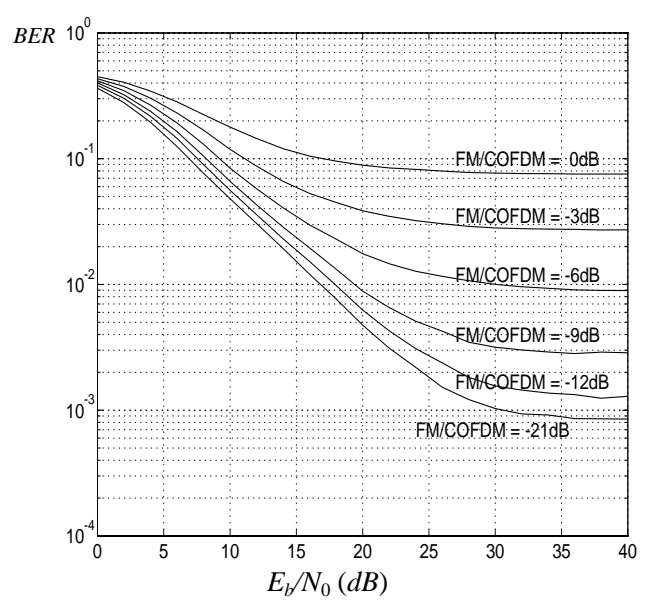

Fig. 9. Performances of the system in a mobile radio channel (without interleaving).

dent. However, it is possible to select the interleaving depth so as to minimize the degradation due to imperfect interleaving. Several studies [14] have been made on the degradation due to a partial interleaving in the case of mobile radio channels.

\section{CONCLUSION}

In this paper, we have analyzed the performances of a mobile radio transmission system based on the power multiplexing of two signals, a commercial broadcasting FM signal and a digital COFDM signal.

The performance of this system for different propagation conditions were evaluated using Monte Carlo simulations. The transmission channel was modeled with timevarying impulse responses obtained using the time-frequency scattering equation of the channel. With this approach, both time and frequency variations of the channel were taken into account, as well as the physical mechanisms influencing the propagation through the channel.

From the results that we obtained, we can conclude that the performances of the COFDM system are greatly influenced by the quality of the FM demodulator. In fact, we have shown that the presence of a residual FM signal at the input of the COFDM demodulator could, for large signal to noise ratios, result in an irreducible BER, even when there is no fading. Of course, this effect is even stronger when fading is present. As can be expected, interleaving, in both time and frequency, is very important since it tends to mitigate the effect of deep fades.

\section{REFERENCES}

[1] I. Bruyland, "The Influence of finite bandwidth on the capture effect in FM demodulators," IEEE Transactions on Communications, vol. COM-26, no. 6, pp. 776-784, June 1984.

[2] L. Leentvaar and J. H. Flint, "The capture effect in FM receivers," IEEE Transactions on Communications, vol. COM-24, pp. 531-539, May 1976.
[3] Kintel Technologies Inc., "Digital Audio Broadcasting Using a Compatible System," March 1992.

[4] B. Le Floch, R. Halbert-Lassale and D. Castelain, "Digital Sound Broadcasting to Mobile Receivers," IEEE Transactions on Consumers Electronics, vol. 35, pp. 493-503, August 1989.

[5] M. Leclerc, P. Scalart, P. Fortier, and H. T. Huynh "Performances Analysis of an In-Band COFDM / FM Digital Audio Broadcasting System,"'in Proc. Canadian Conference on Electrical and Computer Engineering, September 1993.

[6] A. P. Bello and B. D. Nelin, "The effect of Frequency Selective Fading on the Binary Error Probability of Incoherent and Differentially Coherent Matched Filter Receivers," IEEE. Transactions on. Communication Systems, vol. CS-11, pp. 170-186, June 1963.

[7] D. C. Cox and R. P. Leck, "Correlation Bandwidth and Delay Spread Multipath Propagation Statistics of $910 \mathrm{MHz}$ Urban Mobile Radio Channels," IEEE Transactions on Communications, vol. COM-33, pp. 1271-1280, November 1975.

[8] R.L. Mickelson, K. C. Yeh, P. E. Argo and T. J. Fitzgerald, "A Rayleigh Fading Channel Simulator," in Proc. 7th International Ionospheric Effects Symposium, May 1993.

[9] W.C. Lee, Mobile Communications Engineering, New York, McGraw-Hill, 1982.

[10] Cost-207, Digital Land Mobile Radio Communications, Final Report 1989, EUR 12160 EN, Commission European Community, Luxemburg.

[11] M. Lecours, J. Y. Chouinard, G. Y. Delisle, and J. Roy, "Statistical Modeling of the received signal envelope in a Mobile Radio Channel," IEEE Transactions on Vehicular Technology, vol. 37, pp. 204-212, November 1988.

[12] R. H. Clarke, "A Statistical Theory of Mobile-Radio Reception,” Bell System Technical Journal, vol. 47, pp. 957-1000, June-August 1968.

[13] M. J. Gan, "A power Spectral Theory of Propagation in The Mobile-Radio Environment," IEEE Transactions on Vehicular Technology, vol. 41, pp. 27-38, February 1972.

[14] F. Gagnon and D. Haccoun, "On the Performances of Error Control Coding with Diversity for Mobile Channels," IEEE Transactions on Vehicular Technology, vol. 41, no. 4, pp. 488495, November 1992.

Pascal Scalart was born in Concarneau, France, in 1965. He received a Doctoral degree in Electrical Engineering from Université de Rennes I, France, in 1992. In 1993, he did postdoctoral work at Laval University, Québec, Canada. His research interests include signal processing for telecommunications.

Michel Leclerc received his B.Sc. degree and his M.Sc. degree in Electrical Engineering from Laval University, Québec, Canada, in 1992 and 1994, respectively. He is now with Cybectec, Québec, Canada. His research interests include design and implementation of realtime signal processing algorithms.

Paul Fortier was born in Québec City, Canada, in 1959. He received his B.Sc. degree and his M.Sc. degree in Electrical Engineering from Laval University, Québec, Canada, in 1982 and 1984, respectively and his M.S. degree in Statistics and his Ph.D. degree in Electrical Engineering from Stanford University in 1987 and 1989, respectively.

Since 1989 he has been at Laval University where is now Associate Professor in the Department of Electrical and Computer Engineering. From 1991 to 1996, he was director of the Computer Engineering program at Laval University. He is now Chairman of the Department of Electrical and Computer Engineering. His research interests include digital signal processing for communications and the study of complexity and performance trade-offs in hardware implementations, 
particularly sub-optimal decoders for TCM and multi-carrier modulation systems.

Huynh Huu Tuê was born in Huê, Viet Nam. He received the B.E.E. and Sc.D. degrees from Laval University in 1966 and 1972 respectively.

Since 1969, he has been on the Faculty of Laval University, Québec, Canada, where he is currently Professor. He was an Invited Professor at the Institut National des Sciences Appliquées, Lyon, France in 1972; Visiting Professor at l'Ecole Nationale des Télécommunications, Paris, in 1980; Invited Professor at the Université de Rennes, France, in 1982; Invited Researcher at The AT\&T Information Systems in Neptune in the summer 1984; Invited Professor at l'Ecole Polytechnique, Montréal, Canada, in 1986; Invited Professor at CEPHAG, France, in 1995; and Invited Professor at INRS-Telecommunications, Montreal, Canada, since 1994.

Dr. Huynh is author and coauthor of several papers in signal processing, computer communications, and communication theory. He has served as consultant to a number of government agencies and industries in the radio-communication domain as well as in signal processing implementation. He is actually in charge of research activities in the field of DSP fast algorithms and architectures with applications to digital communication systems, at the "Laboratoire de Radiocommunication et de Traitement de Signal" at Laval University. 